\title{
Introducing a Novel Chemotherapeutic Drug for the Treatment of Lung Adenocarcinoma: Silver Nanoparticles Green-formulated by Cinnamomum verum
}

\author{
Jianzhong Zhou ${ }^{1}$, Xiaogang Zheng ${ }^{2}$, Qigui Cai ${ }^{1}$, and Chunlin Song ${ }^{3 *}$ \\ ${ }^{1}$ Department of Thoracic and Cardiovascular Surgery, Affiliated Hospital of Jinggangshan University, Ji'an, Jiangxi 343000, CHINA \\ ${ }^{2}$ Department of Emergency, Affiliated Hospital of Jinggangshan University, Ji'an, Jiangxi 343000, CHINA \\ ${ }^{3}$ The Affiliated Hospital of Jinggangshan University, General Medicine, Ji'an, Jiangxi 343000, CHINA
}

\begin{abstract}
In this study we report the green synthesis of nontoxic, stable, and small size silver nanoparticle by Cinnamomum verum with reducing/capping ability without any toxic reducing agents. The in situ prepared AgNPs were characterized by advanced physicochemical techniques like FE-SEM, TEM, and UVVis study. It has been established that AgNPs have a spherical shape with a mean diameter from 10 to 45 $\mathrm{nm}$. In the antioxidant test, the IC50 of AgNPs and BHT against DPPH free radicals were 191 and $242 \mu \mathrm{g} /$ $\mathrm{mL}$, respectively. In the cellular and molecular part of the recent study, the treated cells with AgNPs were assessed by MTT assay for $48 \mathrm{~h}$ about the cytotoxicity and anti-human lung adenocarcinoma properties on normal (HUVEC) and lung adenocarcinoma cell lines i.e. PC-14, LC-2/ad, and HLC-1. The IC50 of AgNPs were 259, 291, and $395 \mu \mathrm{g} / \mathrm{mL}$ against PC-14, LC-2/ad, and HLC-1 cell lines, respectively. The viability of malignant lung cell line reduced dose-dependently in the presence of AgNPs.
\end{abstract}

Key words: Cinnamomum verum, silver nanoparticles, antioxidant, lung cancer

\section{Introduction}

Cancer is recognized as one of the leading causes of death in today's society and several drugs have been introduced to treat this disease, but, most common cancers are not yet controllable and this disease imposes huge costs on the patient and society ${ }^{1-4)}$. The main factor in the development and progression of cancer has not yet been precisely identified; however, the available data suggest that metabolic disorders in the tissue and immune disorders may be involved in the development and exacerbation of this disease. In addition, metabolic disorders in the production and excretion of oxygen free radicals are important factors affecting cancer cells ${ }^{4,5)}$. Free radicals are destructive compounds that are produced as a by-product by the body's chemical reactions and are destroyed by the body's defense system and enzyme system and antioxidants. However, in cases where the body's metabolic disorders and the production of free radicals are high and they are not destroyed by the neutralizing system, due to their instability, these compounds have a strong tendency to react with a variety of molecules in the body ${ }^{6-8)}$. It is estimated that each cell in the human body is exposed to free radicals 10,000 times a day and DNA strands 5,000 times a day. Damage to cell components includes proteins (genetic disorder), fats (lipid oxidation), and cell membranes (permeability disorder) that if the damage is not repaired, it leads to disruption of the chemical reaction and normal proteinization of the cell and the formation of harmful compounds and sometimes cancer cells in the body [8-10]. It is reported that thousands of cancer cells are produced daily in the human body that are killed by the body's defense system. In some cases, due to dysfunction of the above systems, cancer cells proliferate and conditions for cancer development in different tissues ${ }^{7-9)}$. According to the above, antioxidants play a vital role in preventing disorders caused by the effects of free radicals and thus the prevention and treatment of cancer. Antioxidants are a wide range of molecular compounds with complex properties that combine with and neutralize free radicals. The results show that more than 60,000 types of molecular antioxidants have

*Correspondence to: Chunlin Song, The Affiliated Hospital of Jinggangshan University, General Medicine, Ji'an, Jiangxi 343000, CHINA

E-mail: song380316133@163.com

Accepted November 9, 2021 (received for review October 16, 2021)

Journal of Oleo Science ISSN 1345-8957 print / ISSN 1347-3352 online

http://www.jstage.jst.go.jp/browse/jos/ http://mc.manusriptcentral.com/jjocs

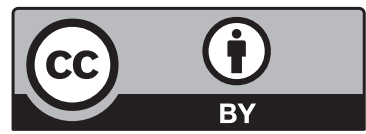


been identified so far. Antioxidants can be effective in three known ways to prevent and treat cancer; 1 . Destruction of free radicals 2. Strengthen the immune system to destroy cancer cells. Prevent the adhesion of cancer cells to other cells and prevent their proliferation ${ }^{6-10)}$.

Today, nanoparticle technology has made great strides in the production of many drugs, and the production of nanoparticles is one of the hopes in the effective treatment and diagnosis of many diseases, including cancer ${ }^{11-14)}$. Metallic nanoparticles have long been considered as a candidate for cancer treatment. Because natural metal oxides are present in large quantities in nature, the processing and synthesis of these nanoparticles can be one of the least expensive synthesis protocols ${ }^{14-17)}$. Metallic nanoparticles are one of the new types of widely used mineral particles that have been considered by researchers due to their suitable physical and chemical properties and at the same time, it has more adsorption power than other metallic nanoparticles-containing compounds ${ }^{12-15}$ ). Metallic nanoparticles are one of the therapeutic compounds recognized by the US Department of Food and Drug Administration as a safe substance. Metallic nanoparticles are biocompatible and non-toxic and have also been used as medical fillers, cosmetics and drug carriers ${ }^{11-13)}$. The special properties of metallic nanoparticles are high catalytic activity, low dielectric constant, high chemical stability, absorption of infrared and ultraviolet light and most importantly its antibacterial properties. If the therapeutic and anticancer effects of these compounds are confirmed, this could be a significant step in advancing cancer therapies ${ }^{14-17}$.

In the present work, we report a simple, environmentally friendly, cost-effective and highly reproducible green synthetic approach for producing Ag NPs using Cinnamomum verum as the stabilizing and reducing agent and water as the dispersion medium. The prepared AgNPs were characterized and applied as efficient anti-human lung adenocarcinoma agent.

\section{Experimental}

Dimethyl sulfoxide(DMSO), Antimycotic antibiotic solution, hydrolysate, decamplmaneh fetal bovine serum, Ehrlich solution, 4-(Dimethylamino) benzaldehyde, 2,2-diphenyl-1-picrylhydrazyl (DPPH), carbazole reagent, boraxsulphuric acid mixture, Dulbecco's Modified Eagle Medium (DMED), and phosphate buffer solution(PBS) all were achieved from Sigma-Aldrich company of USA.

\subsection{Preparation of the AgNPs}

Fresh leaves of Cinnamomum verum were collected, washed several times with distilled water to eliminate the dirt particles present on the leaves. The cleared leaves were dried in the oven at $60^{\circ} \mathrm{C}$ for $12 \mathrm{~h}$ to remove the re- maining moisture, and then ground into powder. The Cinnamomum verum leaf aqueous extract was prepared by adding $20 \mathrm{~g}$ of dried leaves powder in $500 \mathrm{~mL}$ of boiling distilled water for $5 \mathrm{~min}$. Then the mixture was centrifuged at $5000 \mathrm{rpm}$ for $15 \mathrm{~min}$ in a Jouan-type centrifuge, and the supernatant was filtered in flasks to obtain a cell aqueous extract and stored at $4^{\circ} \mathrm{C}$ in refrigerator for further use ${ }^{18)}$.

The green synthesis of the silver nanoparticles was initiated with a reaction mixture of $100 \mathrm{~mL}$ of silver salt $\left(\mathrm{AgNO}_{3}\right)$ in the concentration of $1 \times 10^{-3} \mathrm{M}$ and $200 \mathrm{~mL}$ of aqueous extract solution of $A$. sativum leaf $(20 \mu \mathrm{g} / \mathrm{mL})$ in the proportion 1:10 in a conical flask.

The reaction mixture was kept under magnetic stirring for $12 \mathrm{~h}$ at room temperature. At the end of the reaction time, the black colored colloidal solution of Ag was formed. The mixture was centrifuged at $10000 \mathrm{rpm}$ for $15 \mathrm{~min}$. The precipitate was triplet washed with water and centrifuged subsequently $^{18)}$.

For analyzing silver nanoparticles, the common techniques of organic chemistry, i.e. UV-Vis. spectroscopy, FE-SEM, and TEM were used.

Silver nanoparticles were primarily confirmed using UV-Vis spectroscopy at a scan range from 250-700 nm wavelength (Jasco V670 Spectrophotometer).

The morphological features of silver nanoparticles in terms of surface, shape and sizes were exactly analyzed by common morphological testes i.e., FE-SEM (Fe-SEM ZEISS EVO18) and TEM(TEM FEI-TECNAI G2-20 TWIN)microscopic techniques.

\subsection{Anti-human lung adenocarcinoma properties of Ag- NPs}

The process of the controlled culture of prokaryotic or eukaryotic cells in a filtered or unfiltered flask or cell culture plate by a suitable culture medium is called. This term is mostly used for culturing multicellular cells. Special culture media are used to culture cells. The cells are usually cultured at $37^{\circ} \mathrm{C}$ in equipment such as $\mathrm{CO}_{2}$ incubators. Cell culture should be performed under aseptic (disinfected)conditions because the growth of these cells is much slower than the growth of bacteria and yeasts and there is a possibility of contamination of the culture medium. Antibiotics such as penicillin, streptomycin, or gentamicin are sometimes used to stop the growth of bacteria. In order for cells to proliferate well in culture medium, their density in culture medium must be low. For this purpose, the cells should be passed to the fresh culture medium from time to time. One of the goals of cell culture is to study cells in terms of how they grow, their nutritional needs, and the reasons they stop growing, each of which can have a profound effect on the morphology of the cells we see under a microscope. Therefore, to study the cell growth cycle, develop methods to control the growth of cancer cells and modulate the expression of genes, it is 
necessary to cultivate these cells in the external environment $^{18)}$

With the help of cell culture, cells can be prepared that are in different stages of differentiation and can be differentiated into other cells with the help of hormones and growth factors. With the help of cell culture, homogenous cells can be prepared and intracellular activities such as DNA replication, DNA transcription synthesis, RNA and protein synthesis and other details related to metabolism can be studied. It is also possible to examine the subsequent events and intracellular currents, such as the displacement of these complexes, the type of intracellular messages, and how the messages are transmitted, after connecting different molecules to the corresponding membrane receptor. The cultured cells can be stored frozen at very low temperatures. Such conditions will maintain the growth rate or genetic composition of these cells and can be thawed and used again at the appropriate time. This prevents the aging of cells, while it is currently not possible to prevent the aging of animals. When working with laboratory animals, systemic changes due to the effect of the animal's natural homeostasis or the stress of the experiments on the results should be considered. While the use of cell culture eliminates this problem. In addition, standardizing laboratory tests is easier and more practical than tests on living organisms. In laboratory environments, it is much easier to control the physical and chemical factors in the living environment of cells, including acidity, heat, osmotic pressure, and the pressure of gases such as oxygen and carbon dioxide. Cells that are taken directly from the individual are known as primer cells and have a limited lifespan. Most cells have a limited lifespan, except for those taken from a tumor. An immortal cell line can proliferate indefinitely by creating a random or targeted mutation (such as artificial expression of the genus and be established as a representative of specific cell types ${ }^{18)}$.

In this study, PC-14, LC-2/ad, and HLC-1 cells were used to evaluate the anticancer effect of AgNPs on cell culture.

In the recent study, the cells were cultured in medium $($ RPMI1640 = Roswell Park MemoryL Institute1640) with $10 \%$ FBS combined with penicillin and streptomycin antibiotics in an incubator containing $5 \% \mathrm{CO}_{2}$ in a flask (T25). After three passages for purification, the cells were used to perform the next steps. Cell count and the number of viable cells were performed with a hemocytometer slide using trypan blue. Evaluation of the cytotoxic effect of the AgNPs was performed by the modified 3-(4,5-dimethylthiazol-2-yl)-2,5-diphenyl-2H-tetrazolium bromide(MTT) colorimetric test. In this method, MTT, which is yellow, is converted to insoluble and formazan purple dye by the dehydrogenase enzymes in the mitochondria of active cells. The adsorption of this compound can be measured after dissolving at 570-540 nm. After two days and covering the flask bottom with cell, the cell layer adhering to the flask bottom was isolated enzymatically using trypsin-EDTA (5\%) (Tetraacetic acid ethylenediamine), after transfer to sterile test tubes, it was centrifuged at $2000 \mathrm{rpm}$ for 10 minutes. The cells were then resuspended in a fresh culture medium with the help of a pasteur pipette and cell suspension $\left(10^{6} \mathrm{~mL} / \mu \mathrm{g}\right)$ was prepared from them. $40 \mu \mathrm{L}$ of this cell suspension (equivalent to $10^{4} \times 4$ cells) was poured into 96-well plate flat-bottomed wells (for cell culture). Then the final volume of each well with $10 \%$ FBS medium reached $200 \mu \mathrm{L}$. The first-row containing cell suspension was considered as negative control (control). After incubation for 18-24 hours to remove cells from the stress caused by trypsinization, the supernatant was removed slowly and carefully, a new medium was added to all rows with different concentrations of the AgNPs (only a new medium was added to the negative and positive control rows), so that the diluted AgNPs with concentrations of $1-1000 \mu \mathrm{g} / \mathrm{mL}$ was added to the third to sixth rows, respectively, the plate was incubated in $\mathrm{CO}_{2}$ for 48, 24 and 72 hours. After the incubation time, the plate was taken out of the incubator and $20 \mu \mathrm{L}$ of MTT (Sigma)was added to all wells, and incubated for 3 hours. The supernatant was then gently removed and $100 \mu \mathrm{L}$ of DMSO was added to the wells and pipetted to dissolve the formazan crystals. The amount of light absorption(OD) according to the intensity of the blue color of formazan at $540 \mathrm{~nm}$ was read by Eliza reader. To convert OD to the percentage of living cells, the following formula was used and the percentage of cell life at each concentration was calculated after 48, 24 and 72 hours $^{18)}$.

$$
\text { Cell viability }(\%)=\frac{\text { Sample A }}{\text { Control A }} x 100
$$

The concentration of the tested compounds that reduced the percentage of cell life by half was considered as IC50 (The half maximal inhibitory concentration) ${ }^{18)}$.

\subsection{Antioxidant activities of AgNPs}

The DPPH molecule has a stable free radical in which delocalization of the spare electron over the molecule, results the molecules do not dimerise. This delocalization results in the deep violet color with a characteristic absorption peak in ethanol solution at $517 \mathrm{~nm}$. When the DPPH solution was mixed with electron donating substance, which leads to the reduced form with color loss. Later, the solution undergoes further reactions and control the stoichiometry, hence reduced (decolorized) the number of molecules of the DPPH by one molecule of the reductant ${ }^{18)}$.

In this experiment, $3 \mathrm{~mL} 0.004 \%$ DPPH solution was mixed with several concentrations of AgNPs for determining antioxidant properties. A group containing $3 \mathrm{~mL}$ ethanol and $3 \mathrm{~mL}$ DPPH was considered as the control group. According to the international standards, the samples absorption rate was determined at $517 \mathrm{~nm}$ and the antioxidant potentials of AgNPs were assessed according to the following formula ${ }^{18)}$ : 
Inhibition $(\%)=\frac{\text { Sample A }}{\text { Control A }} x 100$

\subsection{Qualitative Measurement}

At least three independent replications were performed for each data and the result was presented as mean \pm SD. Data statistical analysis was done with SPSS software version 19 and Anova Way One and Duncan's test. Significance was considered at the level of $p \leq 0.05$.

\section{Results and Discussion}

\subsection{Structural characterization of synthesized Ag NPs}

FE-SEM analysis is one of the common chemistry tests for determining the morphology and size of several materials such as metallic nanoparticles. In the present study, the FE-SEM image of silver nanoparticles synthesized using Cinnamomum verum leaf aqueous extract is shown in Fig. 1. The Ag nanoparticles appeared as an agglomerated structure. The hydroxyl groups present in Cinnamomum verum could be responsible for agglomeration ${ }^{18)}$. Also, FE-SEM images indicated the range size of 16-44 nm and the shape of spherical for gold nanoparticles.

TEM is the other test for determining the morphology and size of metallic nanoparticles. In our study, the range size of the nanoparticles $(12-41 \mathrm{~nm})$ calculated through TEM images (Fig. 2) . Furthermore, the histogram plot from the TEM image showed the particle size distribution of biosynthesized silver nanoparticles ranges of 10 to $45 \mathrm{~nm}$. In the previous studies, the size of silver nanoparticles formulated by aqueous extract of medicinal plants had been cal-

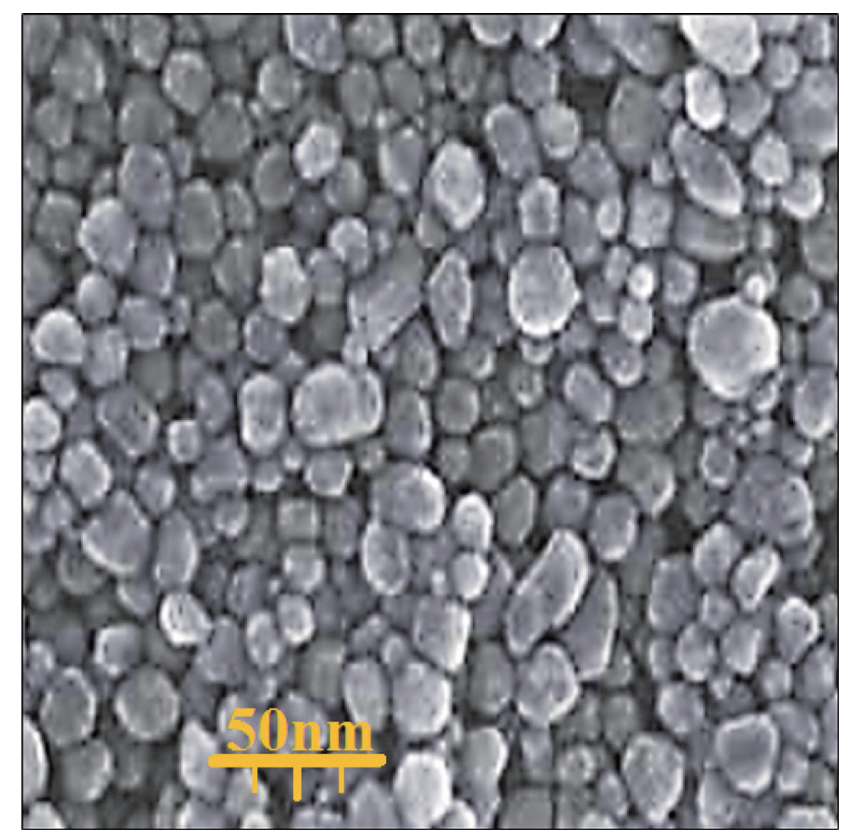

Fig. 1 FE-SEM image of AgNPs.

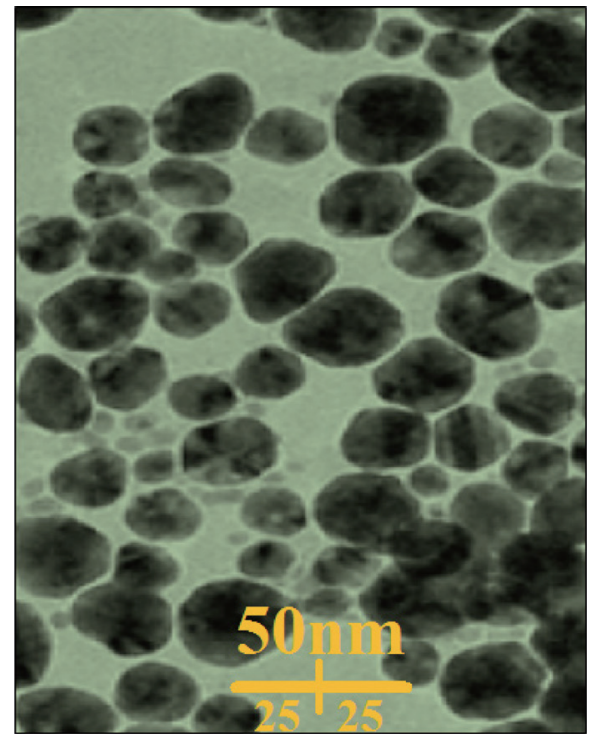

Fig. 2 TEM image of AgNPs.

culated in the ranges of 10-45 nm with the shape of spheri$\mathrm{cal}^{18)}$. These reports support the results of the current work.

Ultraviolet-visible spectroscopy or ultraviolet-visible spectrophotometry (UV-Vis or UV/Vis) refers to absorption spectroscopy or reflectance spectroscopy in part of the ultraviolet and the full, adjacent visible regions of the electromagnetic spectrum. This means it uses light in the visible and adjacent ranges. The absorption or reflectance in the visible range directly affects the perceived color of the chemicals involved. In this region of the spectrum, atoms and molecules undergo electronic transitions. Absorption spectroscopy is complementary to fluorescence spectroscopy, in that fluorescence deals with transitions of electrons from the excited state to the ground state, while absorption measures transitions from the ground state to the excited state.[18] UV-Vis spectroscopic analysis showed the presence of an absorption peak at $446 \mathrm{~nm}$ which confirmed the formation of the silver nanoparticles (Fig. 3).

\subsection{Analysis of the anti-human lung adenocarcinoma po- tentials of Ag NPs}

Cancer, one of the deadliest causes of death in the world, kills more than 7.5 million people annually. Genetic factors and environmental factors are among the most important factors involved in the development of cancer. On the other hand, environmental factors such as diet, lifestyle, geographical conditions, stressors, age and obesity are involved in the incidence of this disease ${ }^{19-23)}$. Clinicians use a variety of methods to diagnose and treat a variety of cancers, the most important of which are chemotherapy, radiation therapy, surgery, and hormone therapy, but the most important way to fight cancer is chemotherapy, which 


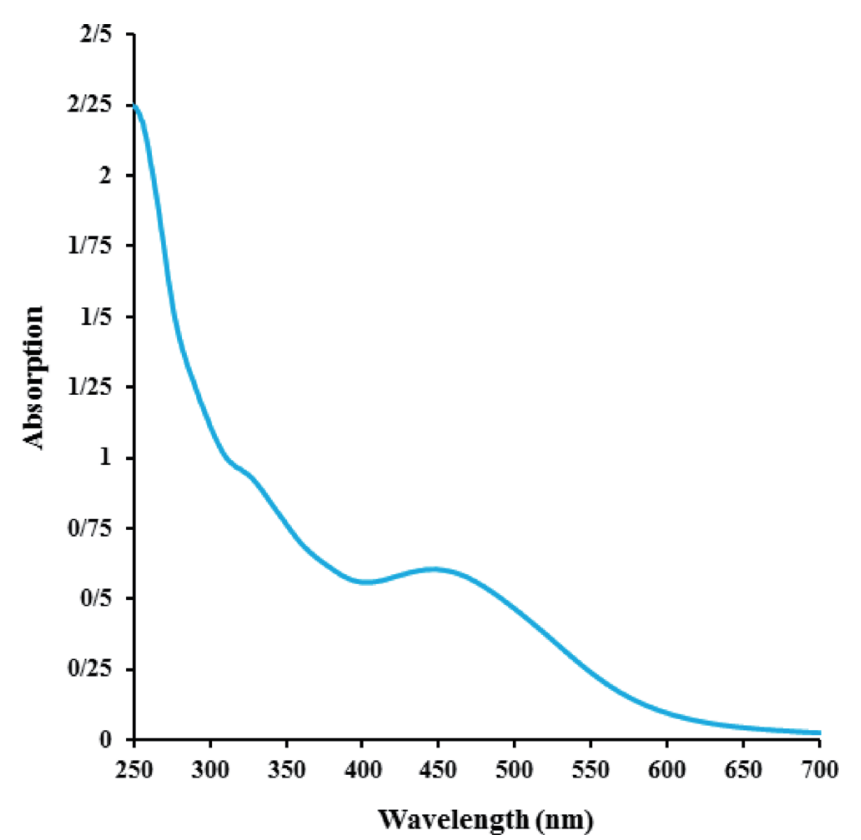

Fig. 3 UV-Vis analysis of AgNPs. causes many side effects for the patient, including fatigue, nausea and hair loss, etc ${ }^{22-26)}$. These complications can have a great effect on the patient's life quality. Because many of the chemical drugs used to treat cancer cause gastrointestinal disorders, kidney damage, etc ${ }^{27,28)}$. Scientists are looking for drugs with fewer side effects than chemical materials, in which natural compounds and molecules have received much attention. Natural compounds and molecules have fewer side effects than chemical materials due to the combination of other compounds with a specific drug effect. Many natural compounds and molecules contain anti-cancer agents that can exert their effects at different stages of the onset and growth of cancer cells ${ }^{29-32}$. The main goal in preventing cancer with natural or chemical substances is to slow down or inhibit the carcinogenic process. This approach focuses purposefully on abnormal intracellular pathways that lead to abnormal cellular function $^{29,33)}$.

In this investigation, the treated cells with different concentrations of the present AgNPs were assessed by MTT assay for $48 \mathrm{~h}$ about the cytotoxicity properties on normal

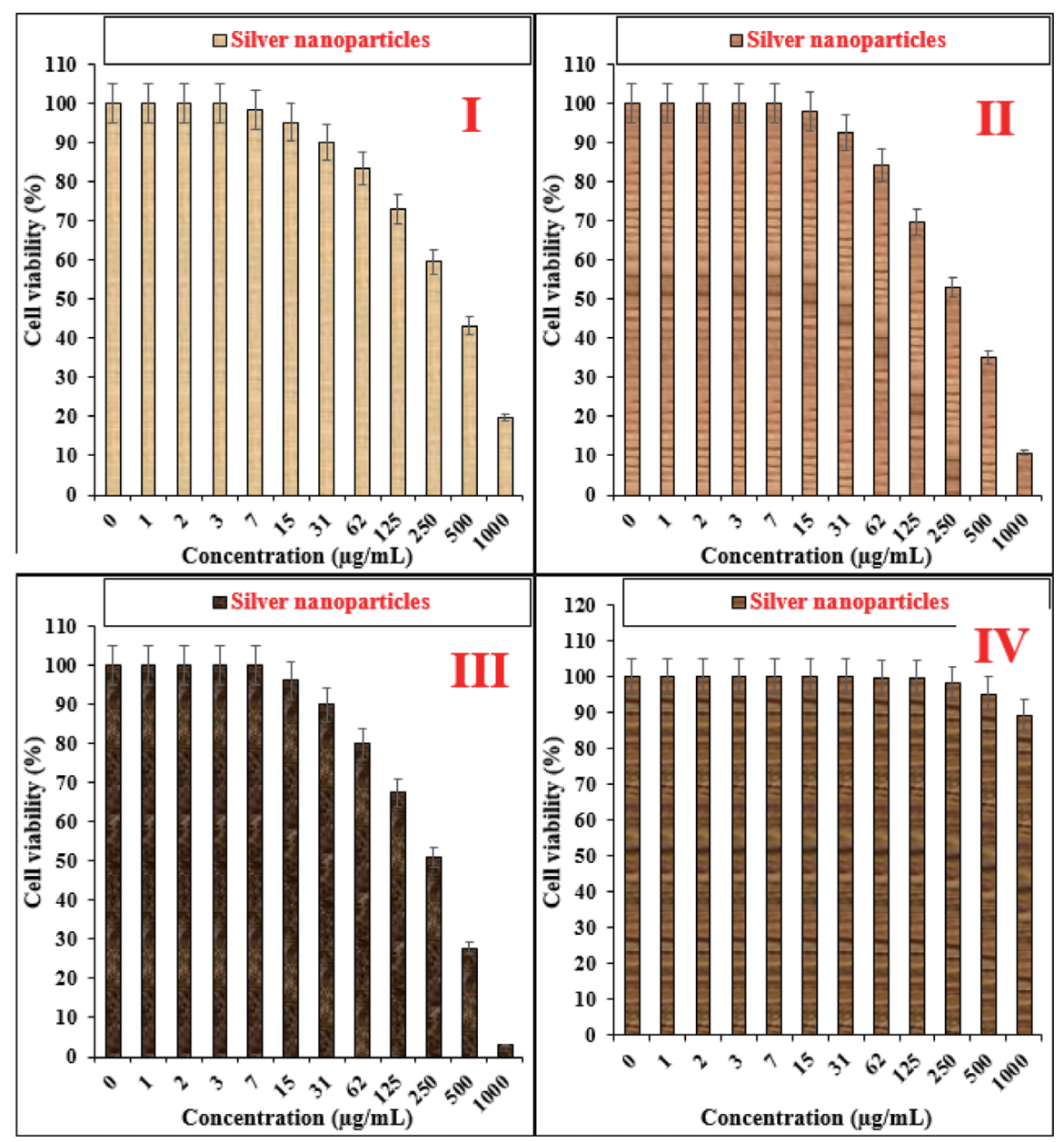

Fig. 4 The cytotoxicity and anti-lung adenocarcinoma properties of Ag NPs against HLC-1 (I), LC-2/ad (II), PC-14 (III), and HUVEC (IV) cell lines. 
Table 1 The IC50 of Ag NPs in the anti-lung adenocarcinoma test.

\begin{tabular}{cc}
\hline & $\begin{array}{c}\text { Silver nanoparticles } \\
(\mu \mathrm{g} / \mathrm{mL})\end{array}$ \\
\hline IC50 against HUVEC & - \\
IC50 against HLC-1 & $395 \pm 0^{\mathrm{b}}$ \\
IC50 against LC-2/ad & $291 \pm 0^{\mathrm{a}}$ \\
IC50 against PC-14 & $259 \pm 0^{\mathrm{a}}$ \\
\hline
\end{tabular}

(HUVEC) and lung malignancy cell lines i.e. PC-14, LC-2/ ad, and HLC-1 (Fig. 4) .

The absorbance rate was evaluated at $570 \mathrm{~nm}$, which represented viability on normal cell line (HUVEC) even up to $1000 \mu \mathrm{g} / \mathrm{mL}$ for AgNPs (Table 1 and Fig. 4).

The viability of malignant lung cell line reduced dose-dependently in the presence of AgNPs. The IC50 of AgNPs were 259, 291, and $395 \mu \mathrm{g} / \mathrm{mL}$ against PC-14, LC-2/ad, and HLC-1 cell lines, respectively (Table 1).

\subsection{Analysis of the antioxidant potentials of AgNPs}

Free radicals are molecules with a free electron ready to react, and oxygen is produced with some molecules. If many of them are suddenly produced in the body, they react with some parts of the cell, such as DNA and cell membranes, and cause cell damage or even death ${ }^{34-39)}$. Normally, the body's defense system neutralizes these harmless free radicals. Antioxidants prevent the spread of oxidation chain reactions. Thus, the strength of an antioxidant formed by the contact of an $\mathrm{H}$ atom with a free radical is due to the effect of an antioxidant on the ease with which this $\mathrm{H}$ atom separates from it. Thus, antioxidants can protect cell membranes and various living compounds against oxidants in small amounts ${ }^{33,34)}$. Numerous biochemical and physiological processes may cause the production of free radicals. Reactive oxygen species (ROS) include free radicals and radical-free forms. When the concentration of ROS increases, it can oxidize macromolecules such as proteins, nucleic acids, and membrane lipids, resulting in cell damage and possibly "cell and tissue destruction $^{131-34)}$. Natural compounds and molecules have two main mechanisms for reducing the concentration of ROS, in other words, natural compounds and molecules reduce the concentration of ROS by producing antioxidants and thus prevent cell damage ${ }^{34,35)}$. Recently, many researchers have paid close attention to natural compounds and molecules and their relationship to their antioxidant properties, and many natural compounds and molecules have been studied for their antioxidant activity ${ }^{31-37)}$.

The scavenging capacity of AgNPs and BHT at different concentrations expressed as percentage inhibition has been indicated in Fig. 5. In the antioxidant test, the IC50 of AgNPs and BHT against DPPH free radicals were 191 and

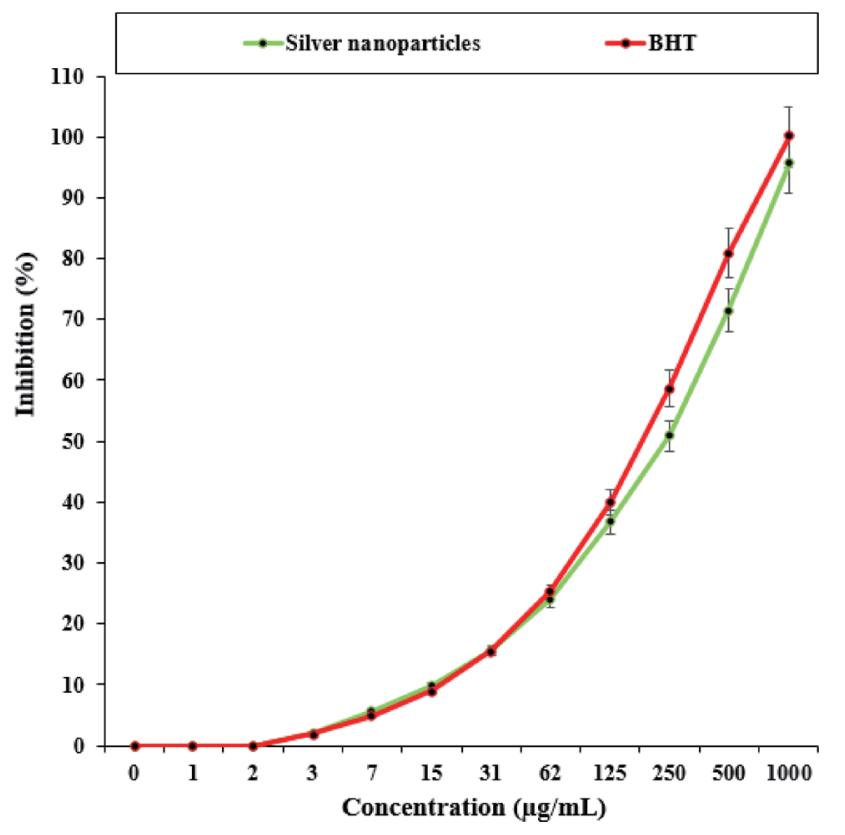

Fig. 5 The antioxidant properties of Ag NPs and BHT against DPPH.

Table 2 The IC50 of Ag NPs and BHT in antioxidant test.

\begin{tabular}{lcc}
\hline & $\begin{array}{c}\text { Silver nanoparticles } \\
(\mu \mathrm{g} / \mathrm{mL})\end{array}$ & $\begin{array}{c}\text { BHT } \\
(\mu \mathrm{g} / \mathrm{mL})\end{array}$ \\
\hline IC50 against DPPH & $242 \pm 0^{\mathrm{a}}$ & $191 \pm 0^{\mathrm{a}}$ \\
\hline
\end{tabular}

$242 \mu \mathrm{g} / \mathrm{mL}$, respectively (Table 2).

\section{Conclusion}

In our research, the gold nanoparticles were successfully obtained from the bioreduction of $\mathrm{AgNO}_{3}$ solutions using an aqueous extract of Cinnamomum verum leaf. The Ag nanoparticles have been appropriately characterized and confirmed using FE-SEM, TEM, and UV-Vis. The viability of malignant lung cell line reduced dose-dependently in the presence of AgNPs. The IC50 of AgNPs were 259, 291, and $395 \mu \mathrm{g} / \mathrm{mL}$ against PC-14, LC-2/ad, and HLC-1 cell lines, respectively. The AgNPs showed the best antioxidant activities against DPPH. After clinical study, AgNPs can be utilized as an efficient drug in the treatment of lung adenocarcinoma in humans.

\section{Author Contributions}

Jianzhong Zhou, Xiaogang Zheng, Qigui Cai, and Chunlin Song had equal roles in designing research and performing research, contributing analytic tools, analyzing data, and 
writing the manuscript.

\section{References}

1) Radini, I.A.; Hasan, N.; Malik, M.A.; Khan, Z. Biosynthesis of iron nanoparticles using Trigonella foenumgraecum seed extract for photocatalytic methyl orange dye degradation and antibacterial applications. $J$. Photochem. Photobiol. B 183, 154-163(2018).

2) Beyene, H.D.; Werkneh, A.A.; Bezabh, H.K.; Ambaye, T.G. Synthesis paradigm and applications of silver nanoparticles (Ag NPs), a review. Sustain. Mater. Technol. 13, 18-23(2017).

3) Chen, X.; Schluesener, H.J. Nanosilver: A nanoproduct in medical application. Toxicol. Lett. 176, 1-12(2008).

4) Alexander, J.W. History of the medical use of silver. Surg. Infect. 10, 289-292 (2009).

5) Bhattacharya, S. Toxicity testing in the 21 century: Defining new risk assessment approaches based on perturbation of intracellular toxicity pathways. PLOS ONE 6, e20887 (2011).

6) Jo, D.H.; Kim, J.H.; Lee, T.G.; Kim, J.H. Size, surface charge, and shape determine therapeutic effects of nanoparticles on brain and retinal diseases. Nanomed. Nanotechnol. Biol. Med. 11, 1603-1611 (2015).

7) Rai, M.; Kon, K.; Ingle, A.; Duran, N.; Galdiero, S.; Galdiero, M. Broad-spectrum bioactivities of silver nanoparticles: The emerging trends and future prospects. Appl. Microbiol. Biotechnol. 98, 1951-1961 (2014).

8) Riehemann, K.; Schneider, S.W.; Luger, T.A.; Godin, B.; Ferrari, M.; Fuchs, H. Nanomedicine-- challenge and perspectives. Angew. Chem. 48, 872-897 (2009).

9) Huang, Y.; Fan, C.Q.; Dong, H.; Wang, S.M.; Yang, X.C.; Yang, S.M. Current applications and future prospects of nanomaterials in tumor therapy. Int. J. Nanomed. 12, 1815-1825 (2017).

10) Conde, J.; Doria, G.; Baptista, P. Noble metal nanoparticles applications in cancer. J. Drug Deliv. 2012, 751075 (2012).

11) De Jong, W.H.; Borm, P.J. Drug delivery and nanoparticles: Applications and hazards. Int. J. Nanomed. 3, 133-149 (2008).

12) Borm, P.J.; Robbins, D.; Haubold, S.; Kuhlbusch, T.; Fissan, H. et al. The potential risks of nanomaterials: A review carried out for ECETOC. Particle Fibre Toxicol. 3, 11(2006).

13) Stapleton, P.A.; Nurkiewicz, T.R. Vascular distribution of nanomaterials. Wiley Interdisciplinary Reviews. Nanomed. Nanobiotechnol. 6, 338-348(2014).

14) Patra, J.K.; Das, G.; Fraceto, L.F.; Campos, E.V.R.; Rodriguez-Torres, M.D.P. et al. Nano based drug delivery systems: Recent developments and future prospects.
J. Nanobiotechnol. 16, 71(2018).

15) Itani, R.; Al Faraj, S.A. siRNA Conjugated nanoparticles-A next generation strategy to treat lung cancer. Int. J. Mol. Sci. 20, 6088(2019).

16) Trojer, M.A.; Li, Y.; Wallin, M.; Holmberg, K.; Nyden, M. Charged microcapsules for controlled release of hydrophobic actives Part II: Surface modification by Lbl adsorption and lipid bilayer formation on properly anchored dispersant layers. J. Colloid Interface Sci. 409, 8-17(2013).

17) Liu, D.; Chen, L.; Jiang, S.; et al. Formulation and characterization of hydrophilic drug diclofenac sodium-loaded solid lipid nanoparticles based on phospholipid complexes technology. J. Liposome Res. 24, 17-26 (2014).

18) Lu, Y.; Wan, X.; Li, L.; Sun, P.; Liu, G. Synthesis of a reusable composite of graphene and silver nanoparticles for catalytic reduction of 4-nitrophenol and performance as anti-colorectal carcinoma. J. Mater. Res. Technol. 12, 1832-1843(2021).

19) Thun, M.J.; Hannan, L.M.; Adams-Campbell, L.L.; Boffetta, P.; Buring, J.E. et al. Lung cancer occurrence in never-smokers: An analysis of 13 cohorts and 22 cancer registry studies. PLoS Med. 5, e185 (2008).

20) Taylor, R.; Najafi, F.; Dobson, A. Meta-analysis of studies of passive smoking and lung cancer: effects of study type and continent. Int. J. Epidemiol. 36, 10481059 (2007).

21) Hecht, S.S. Int. J. Cancer 131, 2724-2732(2012).

22) Alsharairi, N.A. The effects of dietary supplements on asthma and lung cancer risk in smokers and nonsmokers: A review of the literature. Nutrients 11, 725 (2019).

23) Guilbert, S.; Gontard, N.; Gorris, L.G. Prolongation of the shelf-life of perishable food products using biodegradable films and coatings. LWT-Food Sci. Technol. 29, 10-17(1996).

24) Miller, K.S.; Krochta, J.M. Oxygen and aroma barrier properties of edible films: A review. Trends Food Sci. Technol. 8, 228-237 (1997).

25) Debeaufort, F.; Quezada-Gallo, J.A.; Voilley, A. Edible films and coatings: Tomorrow's packagings: A review. Crit. Rev. Food Sci. 38, 299-313 (1998).

26) Fazaeli, R.; Aliyan, H.; Fazaeli, N. Heteropoly acid in ionic liquid - An efficient catalyst for the preparation of $2 \mathrm{H}$-indazolo $[2,1-\mathrm{b}]$ phthalazine-triones. Open Catal. J. 3, 14-18(2010).

27) Konda, S.R.; Reguri, B.R.; Kagga, M. Synthesis of 3,4-dihydro-3,3-dimethyl-13-aryl-2H-indazolo [1,2-b] pthalazine-1,6,11 (13H) -triones using tungstated zirconia $\left(\mathrm{WO}_{3} / \mathrm{ZrO}_{2}\right)$. Der Pharma Chem. 6, 228-233 (2014).

28) Celardo, I.; Pedersen, J.Z.; Traversa, E.; Ghibelli, L. Pharmacological potential of cerium oxide nanoparti- 
cles. Nanoscale 3, 1411-1420 (2011).

29) Bhattacharyya, S.; Kudgus, R.A.; Bhattacharya, R.; Mukherjee, P. Inorganic nanoparticles in cancer therapy. Pharm. Res. 28, 237-259(2011).

30) Sau, T.K.; Rogach, A.L.; Jackel, F.; Klar, T.A.; Feldma$\mathrm{nn}, \mathrm{J}$. Properties and applications of colloidal nonspherical noble metal nanoparticles. Adv. Mater. 22, 1805-1825 (2010).

31) Sperling, R.A.; Parak, W.J. Surface modification, functionalization and bioconjugation of colloidal inorganic nanoparticles. Philos. Trans. Ser. A Math. Phys. Eng. Sci. 368, 1333-1383 (2010).

32) Pelaz, B.; del Pino, P.; Maffre, P.; Hartmann, R.; Gallego, M. et al. Surface functionalization of nanoparticles with polyethylene glycol: Effects on protein adsorption and cellular uptake. ACS Nano. 9, 6996-7008 (2015).

33) Day, E.S.; Morton, J.G.; West, J.L. Nanoparticles for thermal cancer therapy. J. Biomech. Eng. 131, 074001 (2009).

34) Namvar, F.; Rahman, H.S.; Mohamad, R.; Baharara, J.; Mahdavi, M. et al. Cytotoxic effect of magnetic iron oxide nanoparticles synthesized via seaweed aqueous extract. Int. J. Nanomed. 19, 2479-2488(2014).

35) Sankar, R.; Maheswari, R.; Karthik, S.; Shivashangari, K.S.; Ravikumar, V. Anticancer activity of Ficus religiosa engineered copper oxide nanoparticles. Mat. Sci. Eng. C 44, 234-239 (2014).
36） Katata-Seru, L.; Moremedi, T.; Aremu, O.S.; Bahadur, I. Green synthesis of iron nanoparticles using Moringa oleifera extracts and their applications: Removal of nitrate from water and antibacterial activity against Escherichia coli. J. Mol. Liq. 256, 296-304(2018).

37) Sangami, S.; Manu, M. Synthesis of green iron nanoparticles using laterite and their application as a Fenton-like catalyst for the degradation of herbicide Ametryn in water. Environ. Technol. Innov. 8, 150163(2017).

38) Beheshtkhoo, N.; Kouhbanani, M.A.J.; Savardashtaki, A.; Amani, A.M.; Taghizadeh, S. Green synthesis of iron oxide nanoparticles by aqueous leaf extract of Daphne mezereum as a novel dye removing material. Appl. Phys. A 124, 363-369 (2018).

39) Radini, I.A.; Hasan, N.; Malik, M.A.; Khan, Z. Biosynthesis of iron nanoparticles using Trigonella foenumgraecum seed extract for photocatalytic methyl orange dye degradation and antibacterial applications. $J$. Photochem. Photobiol. B 183, 154-163(2018).

CC BY 4.0 (Attribution 4.0 International). This license allows users to share and adapt an article, even commercially, as long as appropriate credit is given. That is, this license lets others copy, distribute, remix, and build upon the Article, even commercially, provided the original source and Authors are credited. 